\title{
De la philologie à la sémantique historique en études ottomanes
}

\section{Erdal Kaynar}

\section{(2) OpenEdition \\ Journals}

Édition électronique

URL : https://journals.openedition.org/ejts/7040

DOI : $10.4000 /$ ejts. 7040

ISSN : 1773-0546

\section{Éditeur}

EJTS

\section{Référence électronique}

Erdal Kaynar, « De la philologie à la sémantique historique en études ottomanes », European Journal of Turkish Studies [En ligne], 31 | 2020, mis en ligne le 04 mai 2021, consulté le 16 février 2022. URL : http://journals.openedition.org/ejts/7040 ; DOI : https://doi.org/10.4000/ejts.7040 


\title{
De la philologie à la sémantique historique en études ottomanes
}

\author{
Erdal Kaynar
}

\section{La sémantique historique : faire du neuf avec de l'ancien?}

1 La sémantique historique ou l'histoire des concepts fait partie des courants historiographiques qui jouissent ces dernières années d'une popularité grandissante. Associée en premier lieu à la Begriffsgeschichte mise en place dans le sillage des travaux de l'historien allemand Reinhart Koselleck (1923-2006) principalement dans les années 1970, cette approche connaît désormais une reprise à l'échelle mondiale (SchulzForberg 2014; Steinmetz et al. 2017 ; Pernau 2018). Des chercheurs font valoir l'utilité de la sémantique historique pour étudier l'histoire des géographies non occidentales et répondre aux spécificités de leur passé. L'intérêt pour le langage exprimé dans les documents historiques et son développement au fil du temps constitue désormais une approche à part entière, au-delà d'un regard purement lexical. Une telle histoire suivant le changement de sens des mots semble promettre une approche suffisamment maniable pour être transposée à des domaines divers.

2 Compte tenu de la popularité globale de l'histoire des concepts, il n'est pas étonnant de voir que des interrogations liées à ce courant commencent à se frayer un chemin dans les études ottomanes et moyen-orientales plus généralement. En Turquie même, la référence à Koselleck est désormais fréquente grâce à la traduction posthume de plusieurs de ses textes ${ }^{1}$. Une longue nécrologie de Koselleck constitue probablement le premier texte en turc à présenter le courant historiographique de la Begriffsgeschichte, expression que l'auteur propose de traduire par «kavram-tarih» (Bilmez 2006:23)2. Outre un article récent spécifiquement consacré à une réflexion sur les potentialités d'une histoire ottomane des concepts (Topal, Wigen 2019), plusieurs autres travaux récents ont également fait valoir l'intérêt d'un recours aux méthodes de l'histoire conceptuelle ${ }^{3}$. 
3 Dans la continuité de cet engagement initial, ce numéro propose de discuter l'utilité d'une sémantique historique pour les études ottomanes. Il se veut aussi une mise au point critique, nécessaire à la fois pour préciser les apports possibles d'une telle approche et pour identifier les risques d'une reprise hâtive d'un courant en vogue.

4 Si l'on considère, en première approche, la sémantique historique comme une analyse du langage socio-politique du passé, son objet peut paraitre banal, en particulier dans des disciplines scientifiques à forte inspiration philologique comme les études ottomanes. L'importance accordée aux mots est inscrite dans les études ottomanes à la fois compte tenu de l'intérêt que la langue ottomane en tant que telle a suscité chez les historiens et pour des raisons scientifiques inhérentes à la discipline. Cette importance s'est traduite dans nombre de travaux dont on peut certainement critiquer les présupposés et les approches, mais qui restent des repères. La sémantique historique n'apporte ainsi certainement pas de problématiques entièrement neuves aux études ottomanes; il ne s'agit pas d'affirmer qu'elle s'imposerait pour remédier à un manque d'attention aux mots du passé de la part de chercheurs supposément empêtrés dans des visions « orientalistes $»^{4}$.

5 Dans les faits, la sémantique historique s'inscrit donc dans la continuité de l'importance accordée à la problématique de l'analyse du langage socio-politique dans les études ottomanes ; elle permet de reprendre et de développer cette analyse dans des nouvelles directions. Ces débats méthodologiques et théoriques riches s'articulent avec bon nombre d'interrogations qui marquent le travail de l'ottomaniste, et dont la reprise peut ainsi être fructueuse. En effet, les réflexions méthodologiques et théoriques de la sémantique historique sur le langage en tant que document du passé et élément de l'expérience historique permettent un nouveau regard sur la pratique de l'analyse des mots du passé et peuvent offrir un cadre théorique plus concentré sur le rôle du langage socio-politique en tant que catégorie de l'expérience historique de l'Empire ottoman.

6 Le présent numéro thématique a été préparé dans cette perspective. Les contributions soulignent toutes à leur manière l'utilité de travailler sur les manifestations du langage socio-politique ottoman comme objet privilégié de l'analyse historique. En même temps, elles partent de l'idée qu'un engagement critique avec le courant de la sémantique historique peut pousser plus loin les travaux sur le langage socio-politique ottoman et contribuer ainsi à enrichir les interrogations méthodologiques et théoriques en études ottomanes. En cela, le numéro se veut aussi une contribution aux débats sur la sémantique historique en tant que courant historiographique.

7 Ce numéro propose aussi une mise en garde contre une reprise peu critique de la sémantique historique. Si jusqu'ici, dans les études ottomanes, l'emprunt à la sémantique historique a privilégié la formulation initiale de la Begriffsgeschichte par Koselleck, il serait erroné de la réduire à une histoire des concepts figée dans le temps ${ }^{5}$, d'ignorer les critiques et les développements qu'elle a connus depuis les années 1970 et d'écarter les projets alternatifs auxquels elle a donné lieu parfois depuis les années 1920. Le numéro plaide pour une réception plus ouverte de ce courant dans sa diversité et invite à une souplesse méthodologique. Éviter que la sémantique historique ne devienne une référence simpliste est également nécessaire compte tenu des aléas inhérents à la Begriffsgeschichte elle-même et de son développement historique. Si l'une des promesses d'un travail sur le langage socio-politique du passé est de pouvoir historiciser le sens des mots et souligner leur caractère contingent, la référence aux 
mots du passé a pu se faire et se fait toujours aussi pour réactiver un temps révolu à des fins politiques et suggérer une continuité historique qui mélange le passé, le présent et le futur dans un récit unique de la nation et de son destin transcendantal. La discussion de la sémantique historique se trouve ainsi écartelée entre le passé et le présent, l'analyse des mots devenant l'outil d'une volonté de manipulation langagière (Müller, Schmieder 2016 : 32). Si la sémantique historique permet d'analyser les mots du passé d'une manière critique, elle peut permettre aussi de mettre en garde contre l'instrumentalisation des mots à des fins politiques.

8 Pour mieux discuter l'apport possible de la sémantique historique pour les études ottomanes et être plus précis quant à son objet, cette introduction propose d'abord un aperçu (non exhaustif) de différentes interprétations qu'elle a connues ${ }^{6}$. Elle dresse ensuite un état des travaux existants afin d'identifier de grandes lignes de l'analyse du langage socio-politique en études ottomanes. Elle présente pour finir brièvement les différents textes de ce numéro, en insistant sur leur diversité d'approches, à la lumière des débats sur la sémantique historique.

\section{Qu'est-ce que la sémantique historique?}

9 En linguistique, la sémantique décrit l'étude des rapports entre les signes et leur sens. La sémantique historique désigne, d'une façon générale, la reconstruction des sens des mots à des périodes différentes. Elle s'inscrit clairement dans une problématique diachronique, et se distingue donc des études linguistiques ou philologiques, plus souvent centrées sur la synchronie. Issue de la linguistique qui, depuis l'intervention de Saussure, poursuit largement une conception synchronique des langues comme systèmes de savoir ${ }^{7}$, la sémantique historique s'est développée au cours de la seconde moitié du $\mathrm{XX}^{\mathrm{e}}$ siècle en un courant des études historiques avec des problématiques propres, notamment dans le sillage des travaux de Reinhart Koselleck. En tant que courant historiographique, la sémantique historique étudie le changement de sens dans le langage en lien avec sa pertinence sociale, politique et culturelle. En cela encore, sa démarche diffère de celles de la philologie ou de la linguistique, qui peuvent se contenter d'étudier le changement sémantique entre deux périodes sans en déduire des conclusions relatives au contexte historique.

La désignation principale de ce courant est l'histoire des concepts, ou histoire conceptuelle, traduction du mot Begriffsgeschichte (de même en anglais history of concepts - conceptual history), en référence aux travaux programmatiques de Koselleck. Aujourd'hui « histoire des concepts » et " sémantique historique " sont souvent utilisés comme des formules synonymes, même si la première a tendance à se référer à des études inspirées de la Begriffsgeschichte tandis que la seconde engloberait toute méthode qui étudie le changement de sens dans la diachronie (cf. Müller, Schmieder 2016: 16-18; Steinmetz 2019 : §12).

11 Koselleck pensait l'histoire des concepts comme un élargissement de l'histoire des idées et insistait sur la distinction entre idées et concepts en tant qu'objets de l'analyse historique (ainsi par exemple dans Koselleck 1967b). La différence entre l'histoire des idées et l'histoire des concepts, sujet débattu depuis les années 1960, est devenue en elle-même un objet d'étude historique (Palti 2010 ; Pankakoski 2010) ${ }^{8}$. En tant que méthode d'analyse qui donne le primat à l'historicité, l'histoire des concepts se veut différente de l'histoire des idées qui définirait l'idée comme une entité constante. 
Tandis que «l'idée» se réfère à un objet transhistorique défini par un noyau sémantique stable, se manifestant par des expressions variables selon les périodes et les géographies choisies, le " concept » renvoie à des processus historiques qui donnent à un mot une multiplicité de sens, dont la définition dépend des temps historiques et du contexte socio-politique, de sorte qu'il ne puisse être subsumé par un sens unique. Ainsi, travailler sur les «concepts " plutôt que sur des «idées » promet d'éviter le risque d'anachronisme ou de transposition mécanique d'une problématique normative à un champ d'étude nouveau.

12 Le fait que la sémantique historique se soit développée essentiellement en Allemagne l'inscrit, en tant que courant historiographique, dans la tradition herméneutique et philologique qui a marqué les sciences humaines et sociales allemandes depuis le $\mathrm{XIX}^{\mathrm{e}}$ siècle (Oexle 2009). L'historiographie s'est construite en Allemagne en discussion étroite et dans le prolongement de la philologie, plus développée dans les pays germaniques que dans le reste de l'Europe, avec une insistance particulière sur l'historicité des phénomènes du passé (Boldt 2014; Richter 1995). L'historisme allemand (Historismus - à ne pas confondre avec l'historicisme dans l'espace anglophone) s'est ainsi établi contre l'histoire des idées poursuivie en Angleterre et en France, par exemple par Alexis de Tocqueville ou John Stuart Mill, qui visait à identifier des constantes intellectuelles dans l'histoire pour établir une généalogie des idées libérales. Définissant l'histoire comme un processus de changement et de développement continu, l'historisme faisait valoir que chaque manifestation historique devait être étudiée dans sa logique propre ${ }^{9}$. Cette importance prêtée aux sources était constitutive du développement de la philosophie herméneutique (cf. Iggers 1984 : 133-143, Busse 2015 : 251-254), et l'insistance sur l'acte d'interpréter et de comprendre devait devenir à son tour une inspiration importante de l'histoire des concepts.

\section{La constitution de la sémantique historique en courant historiographique}

13 Telle qu'elle s'est constituée sous la forme de la Begriffsgeschichte, la sémantique historique a fait l'objet d'un nombre important de recherches depuis les années 1990. L'histoire des concepts ayant largement gardé le silence sur sa formation propre (Oexle 2009 ; Müller, Schmieder 2016 : 22-26), il importait de s'intéresser à ses origines sociales et intellectuelles afin de mieux comprendre l'interprétation classique de la sémantique historique, et de faire ressortir l'existence de réflexions parallèles ou divergentes.

Les travaux menés ont permis de voir à quel point l'émergence de l'analyse historique des notions socio-politiques en tant que problématique scientifique, dans les années 1920 et 1930, alla de pair avec une volonté de manipulation sémantique. En effet, les personnages de référence de l'histoire des concepts (Koselleck 1978: 9) appartenaient largement à l'aile conservatrice voire réactionnaire du spectre politique d'alors; leur analyse critique du vocabulaire politique moderne depuis la Révolution française avait pour implication d'établir qu'il s'agissait là d'abstractions idéalistes dangereuses, aliénant l'Homme de sa nature et de son essence ${ }^{10}$. L'objectif était donc double, et mêlait souci d'analyse et désir politique (Kracauer 1977) : d'une part, l'étude critique des notions clés de la vie politique et sociale moderne cherchait à dévoiler leur caractère autoréférentiel et détaché des réalités (tels des termes comme liberté, droits naturels, socialisme ou démocratie); d'autre part, il s'agissait de mettre en avant un 
nouveau langage socio-politique centré sur des concepts plus organiques au service d'un nouveau règne d'ordre national qui allait être incarné dès 1933 par le Troisième Reich (ordre, liberté et droits du Volk, national-socialisme, Führer...). Cette démarche fut poursuivie de façon explicite en droit et en théorie politique par Carl Schmitt, dont les idées eurent une influence majeure sur la théorie des concepts de Koselleck (Pankakoski 2010; Müller 2011 ; Müller, Schmieder 2016 : 209-218).

Le décisionnisme politique de Schmitt était cependant partagé aussi par le médiéviste Otto Brunner qui, mélangeant un souci d'analyse sémantique et d'argumentation politique, lançait en 1937 un programme de « révision des notions clés » au service d'un nouvel «ordre concret» marqué par la domination transcendante du peuple germanique en Europe ${ }^{11}$. Dans ses travaux sur les concepts politiques du Moyen Âge, Brunner suggérait la continuité des notions d'autorité et d'ordre et appelait au retour à un système organique ancien pour mettre fin aux chambardements de la modernité (Algazi 1997 ; Melton 1996). Après la Seconde Guerre mondiale, l'approche sémantique fut reprise par Werner Conze, l'un des historiens les plus influents des années 1950 en République fédérale d'Allemagne, préparant le terrain pour l'émergence de l'histoire des concepts (Müller, Schmieder 2016 : 274-76). Koselleck s'associa dans les années 1960 avec ces deux personnalités pour préparer le projet phare de l'histoire des concepts : le lexique des Geschichtliche Grundbegriffe.

Si l'histoire des concepts s'est développée dans le sillage de travaux qui poursuivaient une analyse sémantique dans le but de construire et éterniser un ordre autoritaire et nationaliste, la réflexion sur les concepts s'inscrivait, plus largement, dans l'effervescence intellectuelle des années 1920, marquée par une expérience de crise et des reconfigurations épistémologiques concernant la définition même de la réalité et du savoir (Gay 1969; Oexle 2007; Karl 2017: 9-10). En Allemagne, les intellectuels conservateurs se trouvaient insérés au sein d'un champ plus large marqué par les travaux de penseurs libéraux ou communistes tels qu'Aby Warburg, Karl Mannheim, Siegfried Kracauer ou Walter Benjamin ${ }^{12}$. Tous ont, à leur manière, réfléchi à la façon de saisir les notions clés de différentes périodes. À rebours des travaux faisant valoir une interprétation conservatrice de la modernité, ces penseurs soulignèrent les tensions sémantiques inhérentes à des concepts (Mannheim 1984), insistèrent sur la fragilité et l'historicité du sens (ainsi Benjamin 1998), et mobilisèrent des approches extralinguistiques pour pousser plus loin l'analyse de l'expression du sens (Kracauer 1994) - des procédés qui n'ont été que redécouverts vers la fin du XXe siècle.

17 La problématique des concepts n'était pas davantage étrangère aux fondateurs des Annales (cf. Richter 1995 : 17; Lüsebrink 2010) : Marc Bloch lança en 1928 son textemanifeste "Pour une histoire comparée des sociétés européennes", appelant à dépasser le provincialisme des historiographies nationalistes pour étudier les cadres de référence conceptuels transfrontaliers qui marquaient le Moyen Âge ${ }^{13}$. Lucien Febvre porta dans ses travaux un intérêt particulier aux "mots ", aux "choses» et à la " parole » en tant que "matière première de l'histoire " (Febvre 1930, 1943). Utilisant des notions telles celles de "collectif mental», de "psychologie historique " ou de "sensibilités ", ces travaux se référaient au langage en tant qu'expérience historique, par-delà la volonté de comprendre les notions socio-politiques principales d'une époque ; ils contribuèrent ainsi à préparer le terrain pour "l'histoire des mentalités " développée à partir des années 1960. Celle-ci, en l'espèce, ne poursuivit pas l'analyse linguistique qu'avaient tentée les fondateurs des Annales; elle n'en devint pas moins à 
son tour une grande inspiration des débats sur la sémantique historique menés à partir des années $1980^{14}$.

\section{Geschichtliche Grundbegriffe et l'interprétation koselleckienne de la sémantique historique}

Koselleck est venu à l'histoire des concepts à la suite d'une étude d'histoire des idées sur les Lumières, Kritik und Krise ${ }^{15}$, lors de ses recherches pour un travail sur l'État prussien et son programme de réforme (Koselleck 1967a). À partir de l'étude d'une masse colossale de documents d'archives et d'écrits des hommes d'État et intellectuels de la première moitié du XIX ${ }^{e}$ siècle, et sous l'inspiration de Conze, il commença à s'intéresser d'une manière systématique au changement sémantique comme objet d'étude propre de la période qu'il appellerait "l'époque charnière» (Sattelzeit) (Koselleck 1967b; 1972 : XV) marquant la disparition du monde ancien et l'émergence du langage socio-politique moderne. Parallèlement à cette recherche, Koselleck ébaucha les contours d'un travail coordonné sur les notions clés de la modernité en coopération avec Brunner et Conze : les Geschichtliche Grundbegriffe. Ce lexique (abrégé habituellement $G G$ ) comporte des entrées pour 122 termes que les directeurs ont identifiés comme notions clés de la langue politique et sociale allemande, principalement du XIX siècle. L'introduction écrite par Koselleck détaille la problématique et la méthodologie du projet (1972; 1967b). Il part du constat que l'historien ne peut comprendre la signification prémoderne des notions clés identifiées sans faire un effort de traduction pour tenir compte du changement élémentaire de sens. Cependant la démarche dépasse clairement le cadre lexical. L'étude de ces notions, décrites à la fois comme « indicateurs » et «facteurs » de la réalité historique, doit permettre, souligne Koselleck, d'accéder à l'expérience historique du réel, et donc de comprendre la transformation de la société entre le $\mathrm{XVIII}^{\mathrm{e}}$ et le $\mathrm{XIX}^{\mathrm{e}}$ siècle, autrement dit sa modernisation ${ }^{16}$.

19 Paru en sept volumes alphabétiques et deux volumes d'index entre 1972 et 1997, le lexique Geschichtliche Grundbegriffe s'est imposé comme un ouvrage de référence ${ }^{17}$. Il est largement consulté, nourrit d'innombrables travaux, et a fortement marqué les sciences sociales en Allemagne, au-delà des études historiques. Aucun courant historiographique allemand ne connaît une notoriété comparable au niveau international, et le projet s'est imposé comme la référence incontournable en sémantique historique, souvent au détriment des projets alternatifs (Pernau 2018: 5-6). Pourtant, bien que les Geschichtliche Grundbegriffe aient été considérés comme une innovation historiographique majeure, différents chercheurs ont poussé dès les années 1970 à une reformulation de leur problématique et de leurs méthodes. Jusqu'à nos jours deux points principaux, théorique et méthodologique, font débat. Premièrement, l'histoire des concepts s'intéresse uniquement à la haute pensée, en négligeant toute forme de pensée plus populaire au profit d'une conception élitiste de la langue dont les contenus essentiels se manifesteraient dans des notions clés. Deuxièmement, et en lien avec le point précédent sur la conception élitiste de la langue, l'attention prêtée à un terme unique néglige des dimensions essentielles de la langue socio-politique, tant au niveau linguistique que matériel. 


\section{Du mot au champ de mots : développement de la sémantique historique et le projet du Handbuch}

20 À partir des années 1980, les interrogations en sémantique historique et les critiques énoncées à l'encontre des $G G$ ont donné naissance à une série de nouveaux travaux. Les interrogations portant sur l'utilité de privilégier des termes uniques ont été un facteur essentiel des réorientations méthodologiques visant à saisir d'une manière plus large la construction du sens au sein du langage socio-politique (Steinmetz 1993; Wienforth 1993). Certaines études ont également tenté de proposer un rapprochement entre l'histoire des concepts et l'histoire intellectuelle de l'École de Cambridge, développée autour de Quentin Skinner et John Pocock notamment, en soulignant l'importance de la construction de sens au sein du contexte discursif des textes (Steinmetz 1993) ${ }^{18}$. En parallèle, la poursuite des catalogages et la constitution de bases de données numérisées de sources historiques (ainsi celle de l'American and French Research on the Treasury of the French Language - ARTFL) ont ouvert de nouvelles possibilités de travail empirique.

21 Parmi les travaux entrepris se détache un autre projet de lexique, centré sur les notions socio-politiques en France à l'époque des Lumières et de la Révolution, dirigé principalement par Rolf Reichardt: le Handbuch politisch-sozialer Grundbegriffe in Frankreich $1680-1820^{19}$. Ce second projet de référence de la sémantique historique allemande ne partage pas l'approche conservatrice de la transformation moderne, et part précisément des deux critiques susmentionnées pour appeler à une révision de l'histoire des concepts et en venir à une définition plus large de la sémantique historique. Une conception moins élitiste de la langue et une analyse plus pointue de son fonctionnement sont censées établir le langage socio-politique en objet central de l'expérience et de l'action humaines. Quoique moins ambitieux que celui des Geschichtliche Grundbegriffe, le projet éditorial du Handbuch a toutefois rencontré d'importants problèmes de financement et de coordination, qui ont à ce jour empêché son achèvement. Il reste mal connu, particulièrement en France, et reste dans l'ombre des Geschichtliche Grundbegriffe à l'échelle mondiale ${ }^{20}$. Néanmoins, il a eu un impact majeur pour l'ouverture méthodologique de la sémantique historique et s'est établi comme une référence des travaux historiques qui mérite d'être approfondie.

Le Handbuch fut conçu dès ses débuts en collaboration étroite avec des historiens de langue française, et s'inspira fortement des courants historiographiques liés à leurs travaux : ainsi de l'histoire des mentalités pensée par Michel Vovelle (Richter 1995 : 83-86), des études lexicométriques d'analyse du discours proposées, par exemple, par Régine Robin et Denise Maldidier et plus tard Jacques Guilhaumou, en association avec la critique de l'idéologie et la notion du discours proposées par le linguiste Michel Pêcheux ${ }^{21}$. Inscrits dans ces courants, le Handbuch porte une attention particulière à l'élaboration de méthodes empiriques visant à arrêter la fréquence et la diffusion des mots. Une autre différence par rapport aux Geschichtliche Grundbegriffe est la variété des sources utilisées, rendue possible par la numérisation avancée des textes de la période révolutionnaire. Pour proposer une analyse sociale en profondeur de la sémantique historique, les auteurs se penchent sur un corpus de documents populaires comme des pamphlets, des catéchismes politiques, des actes notariaux ou des chants. Au cours des années, plusieurs contributeurs ont poussé plus loin la réflexion sur la nécessité d'élargir l'étude du langage en prêtant de plus en plus d'importance à la sémantique 
visuelle. Différents travaux étudient comment des documents iconographiques ont contribué au développement des notions socio-politiques, notamment parce que les sources visuelles se présentent comme des véhicules principaux de l'émergence d'un nouveau vocabulaire politique (et de la mobilisation liée à celui-ci) dans des sociétés majoritairement illettrées ${ }^{22}$.

23 Au niveau méthodologique, le Handbuch privilégie le champ sémantique (nommé aussi champ lexical ou champ de mots) à travers lequel s'exprime un concept ou une série de concepts $^{23}$. Contrairement à ce que laisserait penser le titre de l'ouvrage, la majorité des entrées ne se concentrent pas sur un terme unique mais scrutent une série de mots associés à partir desquels se construisent et se précisent les différents niveaux de sens d'un concept. L'approche par champ sémantique permet également d'inclure des aspects de la sémantique historique qui n'avaient pas été envisagés par l'histoire des concepts originelle: les usages discursifs, les pré-énoncés, les métaphores et les symbolismes associés, ou encore des aspects pragmatiques (se référant à l'utilisateur des signes) comme les situations de communication et la question de l'intention et de l'intentionnalité, chère à l'École de Cambridge. L'approche proposée permet également de s'intéresser aux éléments non textuels de la langue, comme les supports de communication, et d'ouvrir la recherche au champ visuel.

Le Handbuch a également suscité de nouveaux débats sur les conditions matérielles de l'existence de la langue et de sa construction sociale, par-delà les présupposés vagues de l'approche koselleckienne quant aux rapports entre sémantique et histoire sociale. La référence aux courants français de l'histoire sociale, à l'analyse du discours et à la critique de l'idéologie inspirée du marxisme a élargi la conception de la langue comme objet d'étude historique. Celle-ci est essentiellement pensée, dans l'histoire classique des concepts, à partir de l'énoncé - une conception que Koselleck partage, de fait, avec Quentin Skinner comme avec Michel Foucault, sans que les trois auteurs soient d'accord sur l'importance des pratiques communicatives pour comprendre la langue. L'idée que la langue comporte une série d'indices qui constituent un corpus sociohistorique de traces a permis une attention accrue au caractère idéologique de la langue, à la matérialité du sens et aux conflits qui s'y expriment (Pêcheux dir. 1975).

\section{Les cultural studies et leurs parallèles avec la sémantique historique}

En regard de la sémantique historique, une autre approche qui s'est développée en parallèle au Handbuch, voire aux Geschichtliche Grundbegriffe, est celle des cultural studies. Celles-ci, bien que leur objet principal ne soit pas le langage, nourrissent des interrogations sémiotiques qui s'articulent avec différents aspects de la sémantique historique. À leur point de départ, dès les années 1960, les recherches en cultural studies anticipèrent des questions que les auteurs associés au Handbuch seraient amenés à poser ultérieurement : la critique du primat accordé à la haute littérature au détriment des formes d'expression populaires, l'importance du visuel et de l'iconographie politique, le caractère social et matériel de la langue, ou encore l'ambivalence des concepts et les luttes de résistance contre les définitions hégémoniques (Hall et al. 1980). Les cultural studies sont certainement centrées davantage sur les sociétés contemporaines que sur l'histoire, mais font néanmoins valoir un net intérêt pour l'historicité des faits de société. Raymond Williams, considéré comme l'un des 
fondateurs de ce courant, a largement insisté sur l'importance d'étudier les significations des mots de la société contemporaine dans leurs contextes historiques, et a fait valoir des approches qui mettent en lumière les interdépendances entre différentes notions. Cette approche est au cœur de sa définition du concept de "culture ", et plus explicitement encore dans son lexique Keywords, portant sur 131 mots clés de la société et de la culture contemporaines ${ }^{24}$. Au total, les travaux inspirés par les cultural studies montrent une proximité frappante avec les enjeux de la sémantique historique depuis les années 1980, quoique ces similitudes d'approche demeurent peu discutées.

\section{Développements récents en sémantique historique au croisement de l'histoire globale et des humanités numériques}

Les débats plus récents sur la sémantique historique restent vifs au sein des études historiques, et connaissent une diversification théorique, méthodologique et géographique $^{25}$. Perçue jusqu'aux années 1990 comme une niche de l'historiographie germanique, l'histoire conceptuelle est désormais avancée comme la première référence du renom historiographique allemand (Müller, Schmieder 2016 : 392-93). En dehors de l'Allemagne, l'œuvre de Koselleck connaît (au titre de ses réflexions sur le temps) une réception plus importante parmi les philosophes que chez les historiens, par exemple en France où elle a été encouragée par les travaux de Paul Ricœur et de François Hartog. Koselleck y apparaît en penseur des régimes d'historicité, ses apports empiriques d'historien n'étant guère discutés ${ }^{26}$. Ses réflexions méthodologiques n'en demeurent pas moins centrales pour l'ensemble des travaux qui se réclament de la sémantique historique.

La popularité actuelle de cette dernière s'explique, pour partie, par le développement des humanités numériques (cf. Schwandt 2018 ; 2021). D'une part, la numérisation continue des textes a ouvert de nouvelles possibilités à la recherche sérielle. Vérifier l'occurrence d'un mot dans un corpus comprenant des milliers de textes sur plusieurs siècles est désormais une affaire de secondes, ce qui facilite grandement l'analyse des changements de sens dans la diachronie. D'autre part, la déclinaison des champs lexicaux est au cœur des algorithmes utilisés sur internet, et constitue l'outil principal de l'exploitation commerciale des données amassées en ligne. Ainsi la visualisation des champs de mots sous forme de cloud est-elle devenue quotidienne dans les recherches en ligne depuis les années 1990, même si cette visualisation réside de fait sur une approche syntaxique et non pas sémantique.

Dans les dernières décennies, la multiplication des travaux de sémantique historique a permis l'émergence de nouvelles problématiques qui n'étaient traitées que d'une façon accessoire dans les ouvrages classiques de référence. Parmi celles-ci, deux se détachent en particulier. Premièrement, au niveau temporel, l'idée d'élargir la période étudiée. Les travaux de sémantique historique se concentrent essentiellement sur le changement du vocabulaire socio-politique sous l'impact de la modernité, mais ne se passent pas des analyses étymologiques des mots étudiés remontant jusqu'à l'Antiquité grecque, et présentent, souvent en guise de conclusion, une analyse rapide de l'usage de certains concepts jusqu'à nos jours. Des travaux récents appellent à délaisser la 
problématique de la modernisation associée à "l'époque charnière" du XVIII et du XIX siècle, et invitent à s'intéresser aux changements sémantiques des périodes différentes, en particulier ceux du XX $X^{\mathrm{e}}$ siècle ${ }^{27}$.

La deuxième problématique est liée à la reprise de la sémantique historique au-delà de l'historiographie allemande (Pernau 2018: 8-10). Plusieurs historiens se sont inspirés du projet des Geschichtliche Grundbegriffe pour l'adapter aux différentes langues européennes, notamment dans les pays scandinaves et en Espagne (où l'objet d'étude s'étend à pratiquement l'ensemble du monde ibérique). Ce développement a ouvert de nouvelles perspectives sur la question de la traduction et du transfert des concepts entre pays, en lien avec des questions de dialogue interculturel. C'est ainsi que l'histoire conceptuelle évolue désormais à l'échelle européenne, pour en venir à une compréhension des notions qui auraient marqué les pays européens d'une façon transnationale (Steinmetz et al. 2017).

Ces questions ont été reposées aussi par les études sur des géographies non occidentales, qui posent nécessairement la question de l'eurocentrisme méthodologique (Schulz-Forberg 2014; Pernau, Sachsenmaier 2016). Critiques des modèles diffusionnistes d'une migration des idées occidentales vers des pays "périphériques", ces travaux étudient l'émergence du vocabulaire socio-politique moderne comme un processus d'adaptation qui recourt à des registres conceptuels multiples. Plusieurs études discutent ainsi l'apport des transferts non seulement depuis l'Europe mais au sein d'une géographie plus complexe (Park 2012), les nuances du processus d'homogénéisation sémantique à l'échelle mondiale (Sartori 2008) et les façons dont la recherche de nouveaux concepts reflète une tentative de se situer vis-àvis de la restructuration capitaliste du monde (Karl 2017).

\section{Travaux sur le vocabulaire socio-politique en études ottomanes : une sémantique historique « avant la lettre »}

On l'a dit, l'importance prêtée à la langue était inscrite dans la tradition des études ottomanes, à la fois compte tenu du statut historique de l'idiome turc ottoman et pour des raisons disciplinaires. La complexité des institutions ottomanes depuis le $\mathrm{XV}^{\mathrm{e}}$ siècle nécessitait une attention aux termes et au langage de correspondance de la bureaucratie, sur lesquels reposait le fonctionnement de l'administration impériale ${ }^{28}$. De même, la langue se situait directement dans les procédures de légitimité impériale que l'État ottoman poursuivait. D'une part, le développement du turc ottoman en tant que langue de l'État visait à intégrer l'héritage impérial moyen-oriental et islamique à travers une tentative de synthèse entre le turc et les traditions linguistiques de l'arabe et du persan ${ }^{29}$. D'autre part, l'autorité étatique bâtit son pouvoir impérial sur une sémiotique, par le recours à des documents officiels standardisés et la mise en avant d'un langage de pouvoir formalisé censé ordonner le rapport entre sultan et sujets (Ferguson 2018 ; Heinzelmann 2014). Il n'est donc pas possible aux chercheurs de faire abstraction de cette importance accordée à la langue et aux mots, qui compte parmi les faits les plus emblématiques de l'histoire ottomane.

D'autres raisons, tenant à la constitution des disciplines scientifiques, ont motivé l'attention des ottomanistes à l'égard des faits linguistiques. Comme l'ensemble des 
études orientales, les études ottomanes se sont en majeure partie développées à partir de la philologie. La question du déchiffrement de la langue ottomane, la diplomatique des documents écrits forment une tradition savante qui remonte au XVI siècle et qui a été essentielle dans l'établissement des études ottomanes en tant que champ scientifique $^{30}$. À plusieurs égards, la philologie reste aujourd'hui encore leur socle fondamental. Depuis le XIX ${ }^{e}$ siècle et le développement de l'approche historiographique scientifique, cette méthode philologique s'est appliquée non seulement pour scruter la structure immanente d'un ou plusieurs documents, mais aussi pour comprendre l'histoire à travers la langue ottomane (Kreiser 2015). Cette approche ne fut cependant guère théorisée ni systématisée, et a pu s'exposer à la critique d'un certain « fétichisme archivistique » (ou " fétichisme des documents »), donnant à penser que les documents ottomans pouvaient être traités comme le reflet d'une vérité historique, sans s'intéresser à leur langage en tant que source historique propre. En outre, l'orientation philologique des études ottomanes s'est souvent accompagnée d'un primat accordé aux archives de l'État et d'une approche turco-centrique, comme si les documents étatiques en langue turque devaient offrir l'unique accès à la connaissance du passé ottoman ${ }^{31}$.

Compte tenu de l'importance de la méthode philologique en tant qu'outil d'analyse des documents anciens, le développement des travaux sur le vocabulaire socio-politique fut particulièrement marqué s'agissant des périodes antérieures au $\mathrm{XIX}^{\mathrm{e}}$ siècle ${ }^{32}$. On ne saurait en déduire que l'intérêt pour les mots ait été absent des études portant sur le dernier siècle ottoman. L'entrée de l'Empire ottoman dans la modernité globale au $\mathrm{XIX}^{\mathrm{e}}$ siècle fut accompagnée d'une transformation profonde de son langage sociopolitique. L'intérêt que l'État ottoman et l'élite intellectuelle portèrent alors aux faits de langue signale leur conscience de ce que la transformation serait aussi linguistique. Le langage socio-politique élaboré au siècle des réformes, et la recherche de nouveaux mots pour exprimer de nouvelles expériences et attentes, n'ont ainsi pas échappé à l'attention des historiens de la période. En atteste une littérature importante, mobilisant des approches diversifiées.

Plusieurs de ces études privilégient la question de l'impact occidental et de ses conséquences sur le vocabulaire islamique prémoderne. Les plus anciens parmi ces travaux, s'inspirant de la théorie de la modernisation, ont analysé le renouvellement du langage par traduction du vocabulaire socio-politique occidental vers l'ottoman. C'était postuler l'immobilité linguistique de ce dernier, par contraste avec le dynamisme linguistique occidental forçant le changement du langage socio-politique et inculquant l'émergence d'idées et d'institutions nouvelles. Le point de départ de l'analyse était au fond, davantage qu'un concept, une idée dérivée en termes normatifs de l'histoire européenne, dont il convenait d'étudier l'adaptation, nécessairement retardée et incomplète, dans le contexte ottoman ${ }^{33}$. À rebours de cette approche, une littérature plus récente insiste sur les origines endogènes de la modernisation ottomane, donc de l'émergence d'un langage socio-politique moderne. Bien qu'ils récusent l'idée que l'adaptation incomplète des notions intellectuelles occidentales ait été causée par une quelconque insuffisance linguistique du langage classique, ces travaux ont tendance à s'appuyer sur des éléments d'argumentation homologues (Sönmez 2016; Yılmaz 2016; Wigen 2018). Afin de faire valoir que le langage socio-politique prémoderne n'avait rien d'insuffisant, et que sa transformation au XIX ${ }^{e}$ siècle ne fut pas tributaire de l'influence occidentale, ces travaux en viennent à présenter le contenu des notions modernes comme anticipé par la richesse du langage prémoderne. Dans les deux cas, des préconceptions relatives aux continuités et aux ruptures dans l'histoire ottomane 
priment sur l'analyse du changement sémantique entre l'ancien et le nouveau langage ${ }^{34}$.

D'autres études dans le domaine ne s'intéressent à cette question de continuité que d'une façon accessoire et poursuivent une approche diachronique centrée sur le XIX ${ }^{\mathrm{e}}$ et le début du XXe siècle (cf. Reinkowski 2005 : 32-33). Ici, c'est le langage socio-politique lui-même et son évolution au cours du dernier siècle ottoman qui sont l'objet d'étude. Le vocabulaire ottoman est analysé dans son rapport avec les nouvelles conceptions politiques exprimées à différents niveaux de l'État et de la société. La variété des approches adoptées pour ce faire indique assez à quel point la méthode d'analyse dépend du choix du corpus à analyser. Tandis que certains analysent le rapport entre le vocabulaire administratif et les politiques de centralisation à travers des correspondances bureaucratiques (Aymes 2010 ; Reinkowski 2005), d'autres étudient des publications gouvernementales afin de suivre le développement de notions modernes de la politique officielle (Doganalp-Votzi, Römer 2008 ; Heinzelmann 2014); d'autres encore montrent comment l'usage des mots put devenir un instrument de légitimation du pouvoir impérial (Deringil 1998: 21, 39-43), ou donna lieu à des interprétations divergentes de la définition de nouveaux concepts politiques dans certains traités ou périodiques de l'époque (Kieser 2002 ; Zemmin 2018).

Ce n'est que récemment que différents travaux ont commencé à se référer au courant de l'histoire des concepts dans l'analyse du changement sémantique en contexte ottoman. Il est certainement trop tôt pour en faire un bilan mais quelques traits généraux peuvent être relevés. Il y a d'abord une démarche méthodologique commune, revendiquée d'une manière plus explicite que la majorité des travaux précédents. L'ensemble des études partent du principe qu'il faut rester proche des sources et du langage historique ottomans, pour analyser les mots ottomans qui relatent une évolution eux-mêmes, plutôt que d'établir une analyse historique à partir des termes normatifs des langues occidentales exprimant des idées ${ }^{35}$. Dans cette perspective, l'évaluation de ces évolutions est secondaire à leur analyse, ce qui amène les auteurs à prêter une attention accrue à l'importance des notions prémodernes (Yıldız, Gündüz 2019 : 276-278). La caractéristique la plus distinctive des travaux inspirés de l'histoire conceptuelle par rapport à la majorité des travaux précédents, est l'attention prêtée aux différents niveaux de temporalité qui s'expriment dans le vocabulaire sociopolitique ottoman. Ces travaux récusent l'idée d'une périodisation imposée et insistent au contraire sur la nécessité de prendre en considération l'expérience de temporalité des acteurs du passé par l'analyse précise du langage - un procédé qui permet de mettre en cause le départage du temps historique moyen-oriental selon des critères exogènes $^{36}$. Ces études s'inspirent ainsi du volet temporel de l'œuvre de Koselleck et contribuent par cela au renom international de celui-ci comme penseur du temps et de l'historicité (Schulz-Forberg 2013).

\section{Présentation des contributions au numéro thématique}

37 Ce numéro veut contribuer à dépasser une lecture des documents ottomans qui considère les mots comme le simple reflet des vérités historiques sans s'intéresser à eux en tant que sources propres. Les articles réunis soulignent tous à leur manière que l'engagement avec la sémantique historique dans les études ottomanes doit être poursuivi et porté plus loin. Ils ne font pas valoir une approche théorique et 
méthodologique unifiée. Plus que d'un manque de cohérence, cette variété est révélatrice du fait que les auteurs se sont inspirés des interrogations de la sémantique historique sans la définir comme une discipline monolithique. Elle illustre de fait à quel point une méthode flexible est nécessaire pour prendre compte des spécificités de chaque objet d'étude, et aussi pour tenir compte des limites et difficultés réelles que l'on rencontre dans l'étude des mots du passé. Les articles démontrent aussi que l'approche méthodologique doit nécessairement varier en fonction de la nature des documents étudiés. La variété d'approches renvoie également à la panoplie des débats méthodologiques et théoriques qui agitent la sémantique historique depuis des décennies à l'échelle internationale et aux discussions plus récentes sur son application dans les études ottomanes. Si le nom qui revient le plus souvent dans les différents articles est celui de Koselleck, les articles font valoir des méthodes et des problématiques diverses, et traitent de sujets qui ne sont que secondaires dans l'interprétation classique de ce courant. Ainsi ce numéro se distingue de la majorité de travaux d'histoire conceptuelle précédents, qui tendent à se référer principalement au nom de Koselleck au détriment d'approches divergentes et plus récentes.

L'article de Marc Aymes montre que des procédés d'analyse associés à la philologie ottomane peuvent correspondre à des réflexions récentes en sémantique historique sur l'utilité de réaliser des analyses approfondies d'un corpus homogène et réduit pour comprendre comment se réalisait dans les faits le changement sémantique. L'auteur propose de se mettre dans la tête des bureaucrates et de suivre l'acte d'altération linguistique par lequel le langage formalisé de l'administration ottomane de la fin de l'Empire prenait forme. À partir de quelques affaires de faux, en se concentrant sur un échantillon réduit de textes ainsi qu'un nombre limité d'auteurs anonymes, Aymes peut engager une analyse en profondeur des documents. Le travail fait ressortir l'altération matérielle comme acte performatif et matrice de la production de sens dans le langage administratif ottoman. Par cette analyse, l'auteur met en évidence comment le sens s'exprimait dans des constructions phraséologiques tributaires de relations conceptuelles qui dépassaient la somme des mots exprimés, et souligne ainsi la fragilité sémantique des textes sur la durée.

L'article d'Özgür Türesay revisite certaines interrogations essentielles de l'historiographie ottomane sur le XIX ${ }^{e}$ siècle, en même temps que différents débats clés de la sémantique historique. Centré sur les journaux officiels publiés par l'État ottoman de la première moitié du XIX siècle (Tâkvim-i Vekayi et Moniteur ottoman), l'article s'intéresse au langage politique mis en avant par le pouvoir étatique et plus précisément aux mots de la réforme. Faisant valoir une approche inspirée à la fois des débats sur l'histoire du discours et sur la sémantique historique, il élargit l'étude de la rhétorique du pouvoir au-delà de l'analyse de son vocabulaire en mettant en avant son articulation phraséologique et insistant sur l'ancrage pragmatique et matériel du langage. Ainsi il étudie comment l'émergence d'un nouveau contexte de communication devenait constitutif de la production de sens. Cette approche permet à Türesay d'apporter des éléments nouveaux sur les questions du décalage linguistique entre les mots, les concepts et les réalités désignées, questions qui animent la sémantique historique depuis ses débuts et ont une importance clé pour la compréhension du XIX siècle ottoman. Démontrant que le développement des concepts nouveaux pouvait précéder l'émergence des termes pour les exprimer, Türesay situe le langage politique dans une tension constante et évolutive entre 
l'ancien et le nouveau, tension marquée par l'ouverture des mots au futur et leur détachement du passé. Cette approche "onomasiologique " ajoute des éléments de réflexion importants sur l'émergence du langage politique moderne au Moyen-Orient ainsi que sur la question des continuités et ruptures entre le langage politique classique et moderne de l'Empire ottoman.

Darina Martykánová poursuit quant à elle une approche "sémasialogique ». Elle part du mot fenn pour étudier le changement sémantique que ce mot a connu au cours du dernier siècle de l'Empire ottoman pour s'inscrire au sein d'une nouvelle logique de l'élite ottomane marquée par la référence à la science et la technologie. Martykánová se concentre sur l'usage fait de ce mot au sein des institutions étatiques en se référant à une série de documents internes et s'intéresse surtout à son expression consacrée dans la documentation officielle et officieuse de l'État ainsi que dans les noms des institutions étatiques. Cette approche lui permet d'aller au-delà d'une histoire intellectuelle en montrant que l'importance accordée aux sciences s'articulait dans un cadre de référence institutionnel et dépendait des processus de développement de groupes socio-professionnels nouveaux. L'analyse montre comment le changement du mot fenn révélait l'émergence d'une nouvelle épistémologie et se situait dans un nœud de changements sociaux et matériels marqué par le développement technologique et par la diffusion des sciences techniques dans la société ottomane, par un programme de transformation poursuivi par l'État et par le développement de divers processus socioprofessionnels. Martykánová montre ainsi que l'usage changeant de fenn dévoile l'émergence d'une nouvelle logique technocratique qui se cristallisait comme une condition de la modernisation de l'État et de la société ottomans. Son étude fait ainsi la démonstration de l'utilité d'élargir le champ de la sémantique historique au-delà de la sphère sémiotique pour mieux saisir les développements sociaux, institutionnels et matériels qui ont marqué la fin de l'Empire ottoman.

Si les articles de Marc Aymes, Özgür Türesay et Darina Martykánová renvoient à la temporalité exprimée par les mots, celui d'Ömer Köksal propose d'en faire l'objet d'étude principal. Suivant les travaux de Koselleck comme penseur des temps, il se sert de certaines de ses catégories d'analyse comme méthode heuristique pour faire ressortir la complexité et la spécificité des références temporelles dans le langage socio-politique de la dernière décennie de l'Empire ottoman. Centré sur des essais de deux intellectuels importants de la seconde période constitutionnelle, associés généralement à des camps opposés, Köksal établit une conscience de temporalité commune à ces auteurs comme un des vecteurs principaux de la vie intellectuelle à un moment où la disparition de l'Empire leur semblait imminente. Son analyse d'une série de mots pour dire la temporalité et la crise montre que le langage socio-politique de la fin de l'Empire puisait aussi bien dans le répertoire conceptuel musulman qu'occidental. Ceux-ci ne se présentaient cependant pas comme des entités opposées à des sens figés mais au contraire comme des registres entrelacés. Des notions clés des deux registres étaient mobilisées et transformées de manière à faire ressortir une conscience historique marquée par l'expérience de crise et le pronostic d'une disparition imminente de l'Empire ottoman, conscience qui se traduit en même temps par une attente de la chute de la civilisation européenne. Köksal montre à quel point ces notions étaient imprégnées d'une forte tension temporelle où le passé ne prenait sens que dans son rapport ambigu au présent et à l'avenir. Le constat de crise, le 
discours de déclin, le doute sur l'avenir menaient les auteurs à une impasse conceptuelle existentielle.

L'ensemble de ces contributions répondent à leur manière aux défis d'une analyse du langage socio-politique ottoman. Même sans faire valoir une approche théorique coordonnée, elles renvoient à divers débats de la sémantique historique. L'étude de la charge temporelle qui s'exprime dans les mots entre l'ancien et le nouveau, la mise en avant des ambiguïtés et de la précarité de sens dans la durée, l'insistance sur la nécessité de prendre en compte des procédés extralinguistiques de construction de sens, l'attention aux aspects pragmatiques de communication et de performance pour comprendre le langage au-delà des mots exprimés - l'ensemble de ces analyses s'inscrit dans des interrogations poursuivies en sémantique historique depuis des décennies. Les articles de ce numéro soulignent ainsi l'utilité de se référer à des développements que la sémantique historique a connus en tant que courant historiographique depuis au moins les années 1980, et invitent à une histoire des concepts en études ottomanes qui donne une définition large de la sémantique historique.

\section{BIBLIOGRAPHIE}

Algazi, Gadi (1997). ‘Otto Brunner. “Konkrete Ordnung” und die Sprache der Zeit', in Schöttler, Peter éd., Geschichtswissenschaft als Legitimationswissenschaft, 1918-1945, Francfort-sur-le-Main, Suhrkamp, pp. 166-203.

Ayalon, Ami (1987). Language and Change in the Arab Middle East: The Evolution of Modern Arabic Political Discourse, New York, Oxford University Press.

Aymes, Marc (2021). https://journals.openedition.org/ejts/6914

Aymes, Marc (2010). Un Grand Progrès - sur le papier. Histoire provinciale des réformes ottomanes à Chypre au XIX $X^{e}$ siècle, Louvain, Peeters.

Babinger, Franz (1919). 'Die türkischen Studien in Europa bis zum Auftreten Josef von HammerPurgstalls', Welt des Islams 7(3-4), pp. 103-129.

Bauman, Zygmunt (2004). Wasted Lives. Modernity and its Outcasts, Cambridge, Polity.

Benjamin, Walter (1998 [1982]). Das Passagen-Werk, Francfort-sur-le-Main, Suhrkamp.

Bell, David A. (2001). The Cult of the Nation in France. Inventing Nationalism, 1680-1800, Cambridge, MA, Harvard University Press.

Berkes, Niyazi (1964). The Development of Secularism in Turkey, Montreal, McGill University Press.

Blakemore, Steven (1984). 'Burke and the Fall of Language: The French Revolution as Linguistic Event', Eighteenth-Century Studies 17 (3), pp. 284-307.

Bloch, Marc (1928). 'Pour une histoire comparée des sociétés européennes', Revue de Synthèse Historique 46 (20), pp. 15-50.

Boldt, Andreas (2014). The Life and Work of the German Historian Leopold von Ranke (1795-1886). An Assessment of His Achievement, Lewiston, Edwin Mellen Press. 
Braude, Benjamin (1982). 'Foundation Myths of the Millet-System', in Lewis, Bernard; Braude, Benjamin eds., Christians and Jews in the Ottoman Empire: The Functioning of a Plural Society, New York; Londres, Holmes \& Meier Publishers, pp. 69-90.

Brunner, Otto; Conze, Werner; Koselleck, Reinhart eds. (1972). Geschichtliche Grundbegriffe [GG]: Historisches Lexikon zur politisch-sozialen Sprache in Deutschland, Stuttgart, E. Klett.

Busse, Dietrich (2015). Sprachverstehen und Textinterpretation. Grundzüge einer verstehenstheo- retisch reflektierten interpretativen Semantik, Wiesbaden, Springer.

Bilmez, Bülent (2006). ‘Begriffsgeschichte’nin Babası Reinhart Koselleck (1923-2006) ve Tarihbiliminde Yenilik Arayışları', Toplumsal Tarih Yeni Yaklaşımlar 4 (2006), pp. 9-44.

Cwilinski, Sebastian (2015). 'Die Rezeption der Koselleck'schen Begriffsgeschichte in der Türkei', Forum Interdisziplinäre Begriffsgeschichte 4(1), pp. 46-51.

Delacroix, Christian ; Dosse, François ; Garcia, Patrick eds. (2009). Historicités, Paris, La Découverte.

Deringil, Selim (1998). The Well-Protected Domains: Ideology and the Legitimation of Power in the Ottoman Empire, 1876-1909, Londres; New York, I. B. Tauris.

Dictionnaire des usages sociopolitiques (1770-1815), 8 vol., Paris, Champion.

Doganalp-Votzi, Heidemarie ; Römer, Claudia (2008). Herrschaft und Staat: Politische Terminologie des Osmanischen Reiches in der Tanzimatzeit, Vienne, Verlag der Österreichischen Akademie der Wissenschaften.

Faroqhi, Suraiya (1999). Approaching Ottoman History: An Introduction to the Sources, Cambridge, Cambridge University Press.

Febvre, Lucien (1943), 'Parole, matière première de l'histoire', Annales 4(1), pp. 89-91. https:// doi.org/10.3406/ahess.1943.3101.

Febvre, Lucien (1930), 'Les mots et les choses en histoire économique' Annales d'Histoire Économique et Sociale 2(6), pp. 231-234.

Ferguson, Heather (2018), The Proper Order of Things: Language, Power, and Law in Ottoman Administrative Discourses, Stanford, Stanford University Press.

Findley, Carter V. (1980). Bureaucratic Reform in the Ottoman Empire: The Sublime Porte, 1789-1922, Princeton N.J, Princeton University Press.

Gay, Peter (1969). Weimar Culture. The Outsider as Insider, New York, W. W. Norton \& Company. Gordon, Daniel (2004). Citizens without Sovereignty. Equality and Sociability in French Thought, 1670-1789, Princeton, Princeton University Press.

Guilhaumou, Jacques (2005). 'Où va l'analyse de discours : autour de la notion de formation discursive', Marges Linguistiques 9, pp. 95-114.

Gumbrecht, Hans-Ulrich (2002). Vom Leben und Sterben der großen Romanisten: Carl Vossler, Ernst Robert Curtius, Leo Spitzer, Erich Auerbach, Werner Krauss, Munich, Hanser.

Gutman, David (2015). 'Ottoman Historiography and the End of the Genocide Taboo: Writing the Armenian Genocide into Late Ottoman History', Journal of the Ottoman and Turkish Studies Association 2(1), pp. 167-183.

Hagen, Gottfried (2005). 'Legitimacy and World Order' in Karateke, Hakan T.; Reinkowski, Maurus ed., Legitimizing the Order: The Ottoman Rhetoric of State Power, Leyde; Boston, Brill, pp. 55-83. 
Hagen, Gottfried (2003). 'Translations and Translators in a Multilingual Society: A Case Study of Persian-Ottoman Translations, Late Fifteenth to Early Seventeenth Century', Eurasian Studies 2(1), pp. 95-134.

Hagen, Gottfried; Menchinger, Ethan (2014). 'Ottoman Historical Thought', in Duara, Prasanjit; Murthy, V.; Sartori, Andrew eds., Companion to Global Historical Thought, Malden, Wiley, pp. 92-106.

Hall, Stuart et al. eds. (1980). Culture, Media, Language: Working Papers in Cultural Studies, 1972-79, Londres, Hutchinson.

Haller, Rudof (1971), 'Begriff', in Historisches Wörterbuch der Philosophie, Ritter, Joachim; Gründer, Karlfried; Gabriel, Gottfried eds., Bâle, Schwabe, I, pp. 780-785.

Heinzelmann, Tobias (2014). 'The Ruler's Monologue: The Rhetoric of the Ottoman Penal Code of 1858' Die Welt des Islams 54(3-4), pp. 292-321. https://doi.org/10.1163/15700607-05434P02.

Histoire et langage : travaux allemands en lexicologie historique et en histoire conceptuelle (1983). Numéro spécial de la Revue d'Histoire Moderne et Contemporaine 30/2.

Historisches Wörterbuch der Philosophie (1971-2007). Ritter, Joachim; Gründer, Karlfried; Gabriel, Gottfried eds., Bâle, Schwabe. DOI: 10.24894/HWPh.7965.0692.

Höfert, Almut (2004). Den Feind beschreiben. 'Türkengefahr' und europäisches Wissen über das Osmanische Reich 1450-1600, Francfort-sur-le-Main, Campus.

Iggers, Georg G. (1984). The German Conception of History: The National Tradition of Historical Thought from Herder to the Present, Middletown, Wesleyan University Press.

Karl, Rebecca E. (2017). The Magic of Concepts. History and the Economic in $20^{\text {th }}$ Century China, Durham, Duke University Press.

Kieser, Hans-Lukas ed. (2002). Aspects of the Political Language in Turkey, Istanbul, Isis.

Kohle, Hubertus; Reichardt, Rolf (2008). Visualizing the Revolution. Politics and the Pictorial Arts in Late Eighteenth-Century France, Londres, Reaktion Books.

Koselleck, Reinhart (2006). Begriffsgeschichten, Francfort-sur-le-Main, Suhrkamp.

Koselleck, Reinhart (1978). 'Einleitung', in Koselleck, Reinhart ed, Historische Semantik und Begriffsgeschichte, Stuttgart, Klett-Cotta.

Koselleck, Reinhart (1972). ‘Einleitung', in Brunner, Otto; Conze, Werner; Koselleck, Reinhart eds., Geschichtliche Grundbegriffe: Historisches Lexikon zur politisch-sozialen Sprache in Deutschland, Stuttgart, E. Klett, pp. XIII-XXVII.

Koselleck, Reinhart (1967a). Preußen zwischen Reform und Revolution. Allgemeines Landrecht, Verwaltung und soziale Bewegung von 1791 bis 1848, Stuttgart, Klett-Cotta.

Koselleck, Reinhart (1967b). 'Richtlinien für das Lexikon politisch-sozialer Begriffe der Neuzeit', Archiv für Begriffsgeschichte 11, pp. 81-99.

Koselleck, Reinhart (1959). Kritik und Krise. Eine Studie zur Pathogenese der bürgerlichen Welt, Freiburg; Munich, Karl Alber.

Köksal, Ömer (2021). https://journals.openedition.org/ejts/6963

Kara, İsmail (2002). Bir Felsefe Dili Kurmak: Modern Felsefe ve Bilim Terimlerinin Türkiye’ye Girişi, Istanbul, Dergâh.

Kracauer, Siegfried (1994 [1937]). Jacques Offenbach ou le Secret du Second Empire, Paris, Gallimard. 
Kracauer, Siegfried (1977 [1931]). 'Aufruhr der Mittelschichten', in Das Ornament der Masse, Francfort-sur-le-Main, Suhrkamp, pp. 81-105.

Kreiser, Klaus (2015). 'Historiographie de l'Empire ottoman 1', in Georgeon, François ; Vatin, Nicolas; Veinstein, Gilles eds., Borromeo, Elisabetta coll., Dictionnaire de l'Empire ottoman, Paris, Fayard.

Levend, Agâh Sirri (1960). Türk Dilinde Gelişme ve Sadeleşme Evreleri, Ankara, Türk Tarih Kurumu.

Lewis, Bernard (1988). The Political Language of Islam, Chicago, University of Chicago Press.

Lüsebrink, Hans Jürgen (2010). 'Histoire conceptuelle (Begriffsgeschichte)', in Delacroix, Christian ; Dosse, François ; Garcia, Patrick eds., Historiographie. Concepts et débats, Paris, Gallimard, pp. 161-168

Lüsebrink, Hans Jürgen; Reichardt, Rolf (1990). Die Bastille. Zur Symbolgeschichte von Herrschaft und Freiheit, Francfort-sur-le-Main, Fischer.

Lüsebrink, Hans-Jürgen; Reichardt, Rolf (1983). ‘La « Bastille » dans l'imaginaire social de la France à la fin du XVIII ${ }^{\mathrm{e}}$ siècle (1744-1799)', Revue d'Histoire Moderne et Contemporaine 30(2), pp. 196-234.

Mannheim, Karl (1984). Konservatismus. Ein Beitrag zur Soziologie des Wissens, Francfort am Main, Suhrkamp.

Martykánová, Darina (2021). https://journals.openedition.org/ejts/6937

Meinhardt, Helmut et al. (1976). 'Idee', in Historisches Wörterbuch der Philosophie, Ritter, Joachim; Gründer, Karlfried; Gabriel, Gottfried eds., Bâle, Schwabe, vol. 4, pp. 55-134.

Melton, James Van Horn (1996). 'Otto Brunner and the Ideological Origins of Begriffsgeschichte', in Lehmann, Hartmut; Richter, Melvin eds., The Meaning of Historical Terms and Concepts: New Studies on Begriffsgeschichte, Washington D.C., German Historical Institute, pp. 21-33.

Müller, Jan-Werner (2011). 'On Conceptual History', in McMahon, Darrin; Moyn, Samuel eds., Rethinking Modern European Intellectual History, Oxford; New York, Oxford University Press, pp. 74-93.

Müller, Ernst (2020). Das 20. Jahrhundert in Grundbegriffen. Lexikon zur politisch-sozialen und kulturellen Semantik in Deutschland. URL : https://www.zfl-berlin.org/projekt/das-20-jahrhundertin-grundbegriffen.html

Müller, Ernst; Schmieder, Falko (2016). Begriffsgeschichte und historische Semantik: ein kritisches Kompendium, Berlin, Suhrkamp.

Nerlich, Brigitte (1993). 'La sémantique historique au XIX siècle, en Allemagne, en Angleterre et en France', Histoire Épistémologie Langage 15(1), pp. 5-30.

Oexle, Otto Gerhard (2009). 'Berichte und Diskussionen : Begriffsgeschichte - Eine noch nicht begriffene Geschichte', Philosophisches Jahrbuch 116 (2), pp. 381-400.

Oexle, Otto Gerhard (2007). ‘Krise des Historismus - Krise der Wirklichkeit. Eine Problemgeschichte der Moderne', in Oexle, Otto Gerhard ed., Krise des Historismus - Krise der Wirklichkeit, Göttingen, Vandenhoeck \& Ruprecht, pp. 11-116.

Osborne, Peter (1995). The Politics of Time, Londres, Verso.

Palonen, Kari (2004). Die Entzauberung der Begriffe: das Umschreiben der politischen Begriffe bei Quentin Skinner und Reinhart Koselleck, Münster, LIT Verlag. 
Palti, Elías José (2010). 'From Ideas to Concepts to Metaphors: The German Tradition of Intellectual History and the Complex Fabric of Language', History and Theory 49(2), p. 194-211.

Pankakoski, Timo (2010). 'Conflict, Context, Concreteness: Koselleck and Schmitt on Concepts', Political Theory 38(6), pp. 749-779.

Park, Myoung-Kyu (2012). 'Conceptual History in Korea: Its Development and Prospects', Contributions to the History of Concepts 7(1), pp. 36-50.

Pêcheux, Michel dir. (1975). Analyse du discours, langue et idéologies, numéro spécial de la revue Langages 9(37).

Pernau, Margrit (2018). 'Einführung: Neue Wege der Begriffsgeschichte', Geschichte und Gesellschaft 44 (1), pp. 5-28.

Pernau, Margrit; Sachsenmaier, Dominic eds. (2016). Global Conceptual History: A Reader, Londres; New York, Bloomsbury.

Piterberg, Gabriel (2003). An Ottoman Tragedy: History and Historiography at Play, Berkeley; Los Angeles, University of California Press.

Quélennec, Bruno (2020). ‘ " Lumière sur les Lumières » ou « contre-Lumières » ?', Revue d'Histoire des Sciences Humaines 36, pp. 177-197. DOI : https://doi.org/10.4000/rhsh.4809

Reichardt, Rolf (2017). Lexikon der Revolutions-Ikonographie in der europäischen Druckgraphik 1789-1889, 3 vol., Münster, Rhema.

Reichardt, Rolf (1998). 'Light against Darkness: The Visual Representations of a Central Enlightenment Concept', Representations 61, pp. 95-148.

Reichardt, Rolf (1985). 'Einleitung', in Handbuch politisch-sozialer Grundbegriffe in Frankreich 1680-1820, Heft 1, Berlin; Boston, De Gruyter, pp. 39-147.

Reichardt, Rolf (1982). 'Pour une histoire des mots-thèmes socio-politiques en France (1680-1820)', Mots 5, pp. 189-202.

Reichardt, Rolf et al. (1985-). Handbuch politisch-sozialer Grundbegriffe in Frankreich, 1680-1820, Munich, Oldenbourg, De Gruyter, 21 vol. parus sur 30.

Reinkowski, Maurus (2005). Die Dinge der Ordnung. Eine vergleichende Untersuchung über die osmanische Reformpolitik im 19. Jahrhundert, Berlin; Boston, De Gruyter.

Richter, Melvin (1995). The History of Political and Social Concepts. A Critical Introduction, Oxford, Oxford University Press.

Sariyannis, Marinos (2018). A History of Ottoman Political Thought up to the Early Nineteenth Century, Leyde ; Boston, Brill.

Sartori, Andrew (2008). Bengal in Global Concept History. Culturalism in the Age of Capital, Chicago, Chicago University Press.

Schöttler, Peter (1995). 'Mentalities, Ideologies, Discourses. On the "Third Level" as a Theme in Social-Historical Research', in Lüdtke, Alf ed., The History of Everyday Life. Reconstructing Historical Experiences and Ways of Life, Princeton, Princeton University Press, pp. 72-115.

Schwandt, Silke ed. (2021). Digital Methods in the Humanities. Challenges, Ideas, Perspectives, New York, Columbia University Press.

Schwandt, Silke (2018). 'Digitale Methoden für die Historische Semantik. Auf den Spuren von Begriffen in digitalen Korpora', Geschichte und Gesellschaft 44, pp. 107-134. 
Schulz-Forberg, Hagen ed. (2014). A Global Conceptual History of Asia, 1860-1940, Londres, Pickering \& Chatto.

Schulz-Forberg, Hagen (2013). 'The Spatial and Temporal Layers of Global History: A Reflection on Global Conceptual History through Expanding Reinhart Koselleck's Zeitschichten into Global Spaces', Historical Social Research 38(3), pp. 40-58.

Sigalas, Nikos (2012). 'Des histoires des sultans à l'histoire de l'État. Une enquête sur le temps du pouvoir ottoman (XVI ${ }^{\mathrm{e}}$-XVIII ${ }^{\mathrm{e}}$ siècles)', in Georgeon, François; Hitzel, Frédéric (eds.), Les Ottomans et le temps, Leyde; Boston, Brill, pp. 99-128.

Sönmez, Erdem (2016). 'From kanun-ı kadim (ancient law) to umumun kuvveti (force of people): historical context of the Ottoman constitutionalism', Middle Eastern Studies 52(1), pp. 116-134.

Steinmetz, Willibald (2019). 'La sémantique historique : problèmes théoriques et pratiques de recherche', Politika, consulté le 20/12/2019. URL: https://www.politika.io/fr/notice/semantiquehistorique-problemes-theoriques-pratiques-recherche

Steinmetz, Willibald (1993). Das Sagbare und das Machbare. Zum Wandel politischer Handlungsspielräume, England 1780-1867, Stuttgart, Klett-Cotta.

Steinmetz, Willibald; Freeden, Michael; Fernández Sebastián, Javier eds. (2017). Conceptual History in the European Space, New York ; Oxford, Berghahn Books.

Strauss, Johann (2004). 'An den Ursprüngen des modernen politischen Wortschatzes des Osmanisch-Türkischen', Radoslav Katic ic' ed., 'Herrschaft' und 'Staat'. Untersuchungen zum Zivilisationswortschatz im südosteuropäischen Raum 1840-1870. Eine erste Bilanz, Vienne, Verlag der Österreichischen Akademie der Wissenschaften, pp. 197-256.

Suratteau, Jean-René (1984). ‘Sur les travaux des historiens des deux Allemagnes intéressant la Révolution française : Essai d'historiographie comparée et tendances actuelles', Annales Historiques de la Révolution Française 56(255-256), pp. 180-203.

Topal, Alp Eren; Wigen, Einar (2019). 'Ottoman Conceptual History: Challenges and Prospects', Contributions to the History of Concepts 14(1), pp. 93-114.

Vatin, Nicolas (2012). 'Sur l'emploi des mots dans le récit de la prise de Constantinople par les auteurs ottomans du XVe et du début du XVI e siècle', Documents de Travail du CETOBAC 2, pp. 5-14.

Vatin, Nicolas ; Veinstein, Gilles (2003). Le Serail ébranlé. Essai sur les morts, dépositions et avènements des sultans ottomans XIV ${ }^{e}-X I X^{e}$ siècle, Paris, Fayard.

Wienforth, Monika (1993), Monarchie in der bürgerlichen Gesellschaft: Deutschland und England von 1640 bis 1848, Göttingen, Vandenhoeck \& Ruprecht.

Wigen, Einar (2018). State of Translation: Turkey in Interlingual Relations, Ann Arbor MI, University of Michigan Press.

Williams, Raymond (1985). Keywords: A Vocabulary of Culture and Society, Oxford, Oxford University Press.

Yıldı, Aytaç ; Gündüz, Mustafa (2019). 'Maarif: Transformation of a Concept in the Ottoman Empire at the Beginning of the Nineteenth Century', History of Education 48(3), pp. 275-296.

Y1lmaz, Hüseyin (2016). 'From Serbestiyet to Hürriyet: Ottoman Statesmen and the Question of Freedom during the Late Enlightenment', Studia Islamica 111(2), pp. 202-230.

Zemmin, Florian (2019). ‘The Janus Face of Kātib Çelebi: Reflecting on the Ottoman Saddle Period', Turcica 50, pp. 327-354. 
Zemmin, Florian (2018). Modernity in Islamic Tradition, The Concept of 'Society' in the Journal al-Manar (Cairo, 1898-1940), Berlin, Boston, De Gruyter.

Zemmin, Florian ; Sievert, Henning (à paraître). 'The Saddle Period as a Heuristic Tool for Interrogating the Formation of a Multi-Layered Modernity', Contributions to the History of Concepts.

\section{NOTES}

1. Outre le premier livre de Koselleck sur Kritik und Krise (1959), son entrée «Fortschritt» des Geschichtliche Grundbegriffe ainsi qu'un recueil d'articles ont été traduits en turc : İlerleme (trad. Mustafa Özdemir) Ankara, Dost, 2006 et Kavramlar Tarihi. Politik ve Sosyal Dilin Semantiği ve Pragmatiği Üzerine Araştırmalar, Istanbul, İletişim, 2009. Ce travail initial de traduction est retracé dans Cwilinski 2015.

2. La traduction la plus couramment adoptée est désormais kavramlar tarihi.

3. On peut citer Sariyannis 2018; Zemmin 2018; Yıldız, Gündüz 2019. Un numéro spécial « Near Eastern Conceptual History » est en préparation pour Die Welt des Islams sous la direction d'Alp Eren Topal et Florian Zemmin. Sans se limiter aux études ottomanes, ce projet est à plusieurs égards complémentaire du nôtre.

4. Cette critique, centrale dans le texte de Topal et Wigen (2019), empêche ces auteurs de porter plus loin leur discussion sur les spécificités d'une histoire conceptuelle en études ottomanes.

5. Koselleck lui-même a parlé vers la fin de sa vie de son approche d'histoire conceptuelle comme d'une « camisole» $(2006: 86)$.

6. La référence à ce sujet est l'ouvrage de Müller et Schmieder (2016) qui dresse un panorama des différents courants de la sémantique historique en sciences sociales et humaines. (Une traduction anglaise est en préparation.)

7. Sur la sémantique historique comme branche de la linguistique, voir Nerlich 1993.

8. La complexité de la question n'est guère étonnante si l'on tient compte que la recherche de correspondance en différentes langues pour des notions philosophiques telles que «Begriff » et «Idee » est un débat philosophique en lui-même. Cf. Haller 1971 ; Meinhardt et al. 1976.

9. Il reste à noter que, dans l'interprétation des historiens comme Ranke, Droysen et Meinecke, ces manifestations se référaient toujours à l'État et à la nation, qui étaient ainsi conçus comme des catégories transcendantes de l'histoire allemande. Voir l'ouvrage classique de Georg G. Iggers (1984).

10. Algazi 1997 ; Müller 2011. Il s'agit de fait d'une critique conforme à l'idée d'un trouble dans la langue mise en avant par le conservatisme moderne dès 1789. Pour le cas d'Edmund Burke, voir Blakemore 1984.

11. Son appel à la "révision" (Revision der Grundbegriffe), formulé au congrès allemand des historiens de 1937 (Algazi 1997), montre une proximité sémantique nette avec le célèbre article de Carl Schmitt, "Nous repensons les concepts juridiques de fond en comble " (Wir denken die Rechtsbegriffe um), écrit en 1934 en soutien aux réformes juridiques nazies. Comme le montre Oexle (2009: 384-385), ce topos de révision se retrouve d'une manière systématique chez les intellectuels de droite des années 1920 et 1930 et reposait sur une reprise des idées d'un de leurs opposants, le sociologue Karl Mannheim.

12. Un des points forts du livre imposant de Müller et Schmieder sur la sémantique historique est d'avoir reconstitué cette variété souvent négligée des débats des années 1920 et 1930. Constatant l'absence complète de référence aux travaux des auteurs de ce courant dans les travaux de l'après-guerre, les auteurs parlent de « deux cultures de l'histoire des concepts » (2016: 700). Cf. Gumbrecht 2002. 
13. Oexle (2009) engage dans ce sens une comparaison rapide entre les travaux des deux médiévistes Bloch et Brunner.

14. Sur l'absence d'intérêt pour les manifestations langagières dans les travaux relevant de l'histoire des mentalités, voir Richter 1995 : 81-88.

15. Dans Kritik und Krise, publié en 1959 (traduction française 1987, anglaise 1988, turque 2012), Koselleck étudie à partir d'un langage emprunté largement à Carl Schmitt comment la notion de «critique " incorporait en son sein un potentiel de «crise » et causa la «pathogénèse » de la société bourgeoise. L'ouvrage est devenu un classique de la littérature révisionniste sur les Lumières et la Révolution française. Il a fait l'objet de nombreuses études qui le situent dans la transformation $\mathrm{du}$ conservatisme allemand d'après-guerre. Voir, pour un aperçu bibliographique, Quélennec 2020.

16. Koselleck identifie quatre processus sémantiques centraux de l'époque charnière, permettant d'orienter le travail sur les termes identifiés :

- Verzeitlichung (temporalisation ou historicisation): l'inscription des notions dans une conception historique et leur l'ouverture au futur dans le cadre d'une conception dynamique du temps ;

- Demokratisierung (généralisation) : l'utilisation des notions par un nombre croissant de gens ;

- Ideologisierbarkeit et Politisierung (idéologisation et politisation) : la reprise souvent polémique des notions pour la défense de causes idéologiques, et leur autonomisation au sein de processus politiques.

Cette introduction programmatique n'en laissait pas moins en suspens plusieurs présupposés théoriques de son auteur, par exemple sa conception du rapport entre langue et réalité historique, que Koselleck se dispensa de formuler avec clarté (Palti 2010).

17. La notoriété des Geschichtliche Grundbegriffe dépasse celle que connaît le projet parallèle d'analyse sémantique des notions clés de la philosophie Historisches Wörterbuch der Philosophie, complété entre 1971 et 2007 en treize volumes, consultables désormais en ligne. DOI: 10.24894/ HWPh.7965.0692

18. D'aucuns considèrent que le dialogue entre ces deux courants de l'analyse du langage sociopolitique n'a de fait pas eu lieu : Palonen 2004 ; Müller, Schmieder 2016 : 370-71, 392-95.

19. Reichardt et al. 1985-. Des 30 volumes prévus, 21 ont été publiés à l'heure actuelle, sans ordre particulier. L'introduction de Reichardt, plutôt une monographie par sa taille et la densité des réflexions, présente les approches du projet. Pour une présentation en français, voir Reichardt 1982 : 189-202. Cf. Histoire et langage, numéro spécial de la Revue d'Histoire Moderne et Contemporaine paru en 1983. C Voir aussi Richter 1995 (en particulier pp. 79-98) ; Schöttler 1995.

20. Bien que nombre d'historiens français aient contribué au projet, il n'a connu qu'une réception très modeste en France. Les historiens de la Révolution française des années 1980 l'ont, dans leur majorité, ignoré, ou ont préféré déplorer, en référence aux travaux de Gumbrecht, Lüsebrink et Reichardt, la « vogue linguistico-historique qui sévit ici et là » (Suratteau 1984 : 202). La problématique du Handbuch a encore moins suscité l'intérêt de l'école révisionniste, dont les représentants ont privilégié le travail sur les mots dans la lignée d'une histoire des idées classique, à travers le prisme d'une histoire sociale statique. Les références les plus consistantes se trouvent dans les travaux de Jacques Guilhaumou et de Raymonde Monnier, eux-mêmes contributeurs du Handbuch, et co-directeurs (parmi d'autres) d'un projet parallèle, le Dictionnaire des usages socio-politiques (1770-1815), paru en 8 fascicules entre 1985 et 2007.

21. Schöttler 1995 ; Guilhaumou 2005. Cf. Pêcheux dir. 1975.

22. Encore peu développée au début des années 1980 (cf. Lüsebrink, Reichardt 1983; 1990), la sémantique visuelle est devenue centrale dans les travaux ultérieurs de plusieurs initiateurs du Handbuch : Kohle, Reichardt 2008; Reichardt 1998 \& 2017.

23. On mesure l'impact de ces réflexions du Handbuch par exemple dans Bell 2001 et Gordon 2004. 
24. Williams 1985 (version élargie de la première publication de 1976). Pour une comparaison avec les Geschichtliche Grundbegriffe voir Müller, Schmieder 2016 : 703-706.

25. En témoigne une série de publications : Pernau 2018 ; Steinmetz 2019 ; Steinmetz et al. 2017. Voir aussi la revue Contributions to the History of Concepts publiée depuis 2005.

26. Cf. les chapitres différents dans la partie «Le moment Koselleck» dans Delacroix et al. 2009, ainsi que les travaux d'Alexandre Escudier. Cette réception philosophique de Koselleck n'est pas limitée à la France, ni à la philosophie herméneutique. D'autres exemples sont Osborne 1995 ; Bauman 2004.

27. Voir notamment le projet collaboratif sous la direction d'Ernst Müller, Das 20. Jahrhundert in Grundbegriffen. Lexikon zur politisch-sozialen und kulturellen Semantik in Deutschland. URL : https:// www.zfl-berlin.org/projekt/das-20-jahrhundert-in-grundbegriffen.html

28. Benjamin Braude parle dans ce contexte de «term-conscious bureaucracy» (1982:74).

29. "The very texture of the official language clearly tells how the Ottomans saw themselves in relation to the cultural heritage of the entire Islamic Middle East. " (Findley $1980: 9)$ Il est courant de comparer le recours à la tradition linguistique persane et arabe dans la langue turque ottomane au statut que le grec ancien et le latin revêtaient dans les langues de l'Europe occidentale (Strauss $2004: 197)$.

30. Babinger 1919 ; Kreiser 2015. Cet engagement scientifique s'établissait sur la nécessité perçue de mieux connaître $\mathrm{XVI}^{\mathrm{e}}$ siècle pour répondre au "danger turc » à la suite de l'expansion ottomane. Höfert 2004.

31. Piterberg 2003. Cf. Faroqhi 1999 : 1-2. Cette critique du fétichisme archivistique a été reprise plus récemment dans les débats sur les sources pour étudier le génocide des Arméniens : cf. Gutman 2015.

32. Pour quelques exemples, voir Hagen 2003 et 2005 ; Vatin, Veinstein, 2003 ; Sigalas 2012 ; Vatin 2012. Cf. Sariyannis 2018.

33. Voir par exemple l'étude sur la modernisation de langue arabe par Ayalon 1987 ; l'étude de l'ensemble de l'histoire islamique par Lewis 1988 ; ou encore certains passages de Berkes 1964. On trouve des présupposés similaires dans la lecture nationaliste du développement de la langue turque par Levend 1960.

34. Un contre-exemple, en dépit d'une certaine conception essentialiste et homogénéisante des «traditions" islamique et occidentale, est fourni par İsmail Kara, dont les travaux livrent une approche plus nuancée des rapports entre langages classique et moderne au cours de la modernisation des notions-clés de la pensée ottomane et turque aux XIX ${ }^{\mathrm{e}}$ et XX $\mathrm{X}^{\mathrm{e}}$ siècles (voir par exemple Kara 2002).

35. À titre d'exemple, on étudiera ainsi l'évolution des mots maarif et fenn plutôt que d'importer une conception déjà établie des notions afférentes (instruction et éducation, science et technologie), évitant ainsi de construire une analyse eurocentrée de leur développement, nécessairement retardé, dans l'Empire ottoman. Cf. Yıldız, Gündüz 2019 ; voir ici Martykánová 2021.

36. Hagen, Menchinger 2014. Voir notamment la discussion critique de l'utilité du concept de période charnière (Sattelzeit) pour en étude moyennes-orientales proposée par Zemmin (2018 : 177-184 ; 2019 ; aussi Zemmin, Sievert à paraître). 


\section{AUTEUR}

\section{ERDAL KAYNAR}

Université de Strasbourg

kaynar@unistra.fr 